\title{
Interview the Expert: The Societal Experts Action Network (SEAN) COVID-19 Survey Archive, with Gary Langer
}

Eva Aizpurua ${ }^{1}$ (D)

${ }^{1}$ Trinity Research in Social Sciences (TRiSS), Trinity College Dublin

Keywords: repository, sean, covid-19, open-access, survey research, collaboration

https://doi.org/10.29115/SP-2020-0006

Survey Practice

Vol. 13, Issue 1, 2020

In the midst of the COVID-19 pandemic, multiple collaborative initiatives have emerged. In this edition of "Ask the Expert," we discuss one of them: the Societal Experts Action Network (SEAN) COVID-19 Survey Archive, with Gary Langer. We asked him a series of questions regarding the inception of this project, its development over time, as well as the opportunities and challenges associated with it. We truly appreciate him taking the time to respond during this difficult time.

1. Thank you, Gary, for agreeing to this interview. For those readers who might be less familiar with this initiative, could you describe what the Societal Experts Action Network (SEAN) is?

SEAN is a network of social, behavioral, and economic science experts convened by the National Academies of Sciences, Engineering, and Medicine, in collaboration with the National Science Foundation (NSF). Its chief aim is to support policymakers by connecting them with critical research on the COVID-19 pandemic, as well as to share that research with others, including the public at large. SEAN's co-chairs are Bob Groves, one of the leading lights in the survey research field, a former director of the Census Bureau and now provost at Georgetown; and Mary Bassett, former New York City health commissioner, now at Harvard School of Public Health.

\section{What was the thinking behind this project? How did it start?}

Our work is one piece of the larger SEAN effort. Its genesis dates to the third week in March, when two leaders of the NSF's social and economic sciences division, Danny Goroff and Skip Lupia, reached out to a group of survey researchers seeking ideas about ways to support SEAN's mission. Among their questions was how to effectively bring together high-quality social science data on the pandemic. It was a lightbulb moment: Our company operates a cloudbased knowledge management system, PARC, for survey researchers, their stakeholders, and their audiences. It's built for fast and simple text-based search and retrieval of individual survey questions and associated materials - an ideal platform for a one-stop shop for COVID-19 studies. 
The project aims to solve a specific challenge. There's a vast quantity of important survey research being done on the pandemic. To extract maximum value from it, we first need a way to get our arms around it. We do that by collecting, organizing, presenting, and summarizing it for stakeholders across the board.

\section{What have been some of the challenges or hurdles developing this project?}

First, it's a pretty massive task to go through all the surveys we find and try to identify those that support inference. We're doing quick vets, not full-bore methodological reviews, but even then it takes a good deal of effort. Once one has been cleared, the next step is to reach out to the producer, explain our purpose and request supporting materials, which can take some back-andforth discussion.

We then synthesize findings in a concise, digestible format for those who need it quickly. We're producing a weekly summary of COVID-19 surveys released in the previous week, with links back to the source materials. We'd figured on twoto three-page weekly reports. But the volume and range of these studies are so great that our summaries are running eight to ten pages long. We're wrestling with a firehose of data - all of it fascinating material.

One other item, developing awareness of a new resource always is a challenge. In addition to our existing email distribution list, we've been sending out notices via the AAPOR (American Association for Public Opinion Research), WAPOR (World Association for Public Opinion Research), and ESRA (European Survey Research Association) listservs, and we're eager for recipients to share word with their own networks. Delivering access to these materials as broadly as possible is a central goal.

4. Your work, as part of SEAN, provides access to a wide range of materials (datasets, survey questions, results, reports...) on public attitudes, behavior, and experiences related to COVID-19. Who are the intended users of these materials?

I should note that SEAN's efforts are more extensive than the archive alone. As to our corner of SEAN's work, the archive is an open-access resource-no firewalls, fees, or passwords. Anyone with an interest in surveys relating to the pandemic can jump in and have a look at the latest data. That can be an academic researcher investigating a specific question; a federal official, governor, or mayor evaluating a change in policy; a news reporter working on a story; or simply a member of the public who's trying to stay well informed.

5. How can SEAN assist researchers, policymakers, the media, and the general public during the current crisis? 
Our goal is to make the archive component of SEAN as robust as possible for users with a variety of needs and interests. That's why we're looking not only for topline results and analytical reports but the full gamut of associated materials: de-identified datasets for secondary analysis; scripted questionnaires, essential for replication; banner books of cross-tabulated data, for expedited subgroup analysis; full methodological disclosure-and, frankly, anything else we can get and share.

Uses of these materials are as wide-ranging as the data themselves, from policy to public health to economic impacts and more. What are public views on social distancing and approaches to reopening? Who wants testing, and who's getting it? What's the extent of job losses, furloughs, and other economic impacts across groups? How many people are experiencing stress and anxiety, and to what extent? How is the public assessing the responses of government leaders and institutions? What policy preferences exist, with what changes over time? These are among the many key questions our field seeks to answer. The SEAN archive's role is to collect, organize, summarize, and disseminate the findings.

Policymakers with questions for the broader network can contact SEAN@nas.edu.

\section{National and international contributors are participating in this initiative, and the number of contributors keeps growing. Were you expecting so much collaboration from colleagues?}

This has been such a heartening element of the project. Researchers across the nation and the globe have come together to share their materials for the public good. They've set aside proprietary or competitive interests to contribute freely to the knowledge base on public responses to the pandemic. It's a shining example of cooperation and collaboration in the face of this crisis-for me, a moment for pride in our field and admiration for our colleagues. Contributors are recognized on the archive website, and we're truly grateful for their participation. It's produced, as of this moment (May 18, 2020), a collection of nearly 200 surveys from more than 25 countries, 66 datasets, and more than 2,200 individual questions.

\section{If I did a survey on COVID-19, how could I contribute?}

Mindful of the requirements for inference, we're looking for probability-based studies. See our Contributors' Guide and contact us at covid-19@parc.us.com.

8. SEAN is a live project, which has been incorporating new resources over time. What would you like to see in the future? 
While most of the leading U.S.-based survey producers have agreed to contribute at the dataset level, a few have held back, and we'd love to see them come in. We're also keen to keep growing the archive internationally with methodological details and datasets as well.

\section{What do you hope will ultimately come from this?}

Policymakers, health experts, reporters, and the public need to know how the public is reacting to this pandemic; it's a critical element in calibrating policy responses, assessing their uptake, and discerning the route forward. Many excellent studies are being done. Our short-term goal is to provide a way into these essential data.

The longer-term matters as well. We're living in an extraordinary time of drama, upheaval, heroism and loss, rimmed with profound uncertainty. The current work of the survey research community will serve as a foundational element of the historical record of these trying times. Our hope is that the SEAN archive will serve as a lasting repository of this important work.

\section{How can people keep up to date on SEAN?}

To keep up-to-date on the SEAN archive, you can sign up for notice of our weekly summaries here, visit https://covid-19.parc.us.com any time, and reach out to us with comments or questions-they are very welcome-at covid-19@parc.us.com.

For information on the larger SEAN project, visit https://www.nationalacademies.org/our-work/societal-experts-actionnetwork for updates or to submit questions.

\section{Author information:}

Eva Aizpurua

Trinity Research in Social Sciences (TRiSS)

6th Floor Sutherland Centre

Arts Building, Trinity College

Dublin 2, Ireland

Email: aizpurue@tcd.ie

Phone number: +353018963196 